\title{
Study on the Mechanism of Detonation to Quasi-detonation Transition
}

\author{
Yun-Feng Liu $^{\mathrm{a}} *$ and Zong-Lin Jiang ${ }^{\mathrm{a}}$ \\ ${ }^{a}$ Institute of Mechanics, Chinese Academy of Sciences, Beijing 100190, China (*yfliu.Ihd@gmail.com)
}

\begin{abstract}
In this paper, the mechanism of detonation to quasi-detonation transition was discussed, a new physical model to simulate quasi-detonation was proposed, and one-dimensional theoretical and numerical simulation was conducted. This study firstly demonstrates that the quasi-detonation is of thermal choking. If the conditions of thermal choking are created by some disturbances, the supersonic flow is then unable to accept additional thermal energy, and the CJ detonation becomes the unstable quasi-detonation precipitately. The kinetic energy loss caused by this transition process is firstly considered in this new physical model. The numerical results are in good agreement with previous experimental observations qualitatively, which demonstrates that the quasi-detonation model is physically correct and the study are fundamentally important for detonation and supersonic combustion research.
\end{abstract}

Keywords: Detonation, Quasi-detonation, Thermal Choking.

PACS: 47.40.Rs, 47.10.ab, 82.20.Wt, 47.40.Ki

\section{INTRODUCTION}

The unique analytical solution for detonation is the Chapman-Jouguet detonation which propagates with a definite CJ detonation speed. However, a kind of unstable detonation propagating with about 50 percent of CJ detonation speed was frequently observed in experiments, which was called quasi-detonation [1-6]. The experimental observations indicated that the quasi-detonation front has a double-discontinuity structure, which is constituted by a leading shock and a chemical reaction zone, but the interval between the leading shock and the reaction zone is much wider than the induction zone of $\mathrm{CJ}$ detonation. The experiments also showed that the thermodynamic properties of quasi-detonation cannot be interpreted by the classical theory, and the transition from detonation to quasi-detonation is precipitate. Although quasi-detonation was usually obtained in obstacle-filled tubes, it has generalized onedimensional properties and its propagation is quite independent of the details of the flow fields since it could occur even in smooth tubes [7].

From the streak photograph obtained by Wagner [8], it is demonstrated that the trajectory of the steady quasidetonation front is parallel to the $\mathrm{c}+$ characteristics in burnt gases, and the propagation speed is quite close to the sound speed of the burnt gases. Lee [9] referred to this combustion regime as the choking regime. It seems that the thermodynamic parameters may play an essential role in quasi-detonation development and propagation since the sound speed depends only on the gas temperature. Oppenheim [10] derived the locus of the thermo-states downstream of the quasi-detonation front on the hypothesis that the front is of the CJ type, and called this particular locus a Q-curve. Shchelkin and Troshin [11] described the propagation of separate discontinuities with the relevant Hugoniot relationships, and referred to it as a generalized Hugoniot curve. Zhu et al. [7] theoretically analyzed the thermodynamic properties of quasi-detonation, and confirmed that the quasi-detonation is not of the $\mathrm{CJ}$ type, being completely different from $\mathrm{CJ}$ detonation. We were puzzled by the fact that quasi-detonation is not of the CJ type. Being not the CJ type means that the thermodynamic properties of quasi-detonation cannot be analyzed by the classical CJ theory. It also means that quasi-detonation cannot be numerically simulated because the present governing equations of detonation only give solutions of CJ type.

CP1233 The $2^{\text {nd }}$ International ISCM Symposium and The $12^{\text {th }}$ International EPMESC Conference

J. W. Z. Lu, A.Y.T. Leung, V. P. Iu, and K. M. Mok

(C) 2010 American Institute of Physics 978-0-7354-0778-7/10/\$30.00 
We were also puzzled that for the same chemical reaction energy input, the quasi-detonation exhibits a velocity being approximately 50 percent of the $\mathrm{CJ}$ detonation speed. Because the detonation speed is proportional to the square root of specific heat release of reactants, this means that nearly 75 percent of chemical reaction energy does not support the detonation propagation. Thus, the mechanism of quasi-detonation is completely different form CJ detonation, and the study on the mechanism becomes fundamentally important. Edwards et al. [12] suggested that the detonation velocity decrease is due to a partial heat loss. Chue [13] discussed two possibilities of the energy losses and disproved Edwards's suggestion. He argued that a heat transfer from quasi-detonation front to surroundings may exist because the temperature of burnt gases is significantly higher than that of surroundings; however, the heat transfer time scale from the burning gases to the tube wall is several orders of magnitude of the characteristic time associated with the quasi-detonation propagation. Therefore, the energy loss due to heat transfer is negligible. Secondly, the energy loss may be caused by the turbulence that is induced by the rough walls, which can cause the scattering of kinetic energy in detonation propagation direction to fluctuations in other directions. However, the detonation speed is insensitive to such little energy loss because it is only proportional to the square root of the energy. Therefore, the system energy losses cannot be the primary mechanism resulting in the speed decrease of detonation.

The study of Zhu et al. [7] confirmed that the thermodynamic states of the reactants between the leading shock wave and the flame front are not the thermodynamic properties introduced by the leading shock wave. The states of this region are influenced by both the original reactants and the combustion products. They thought that it is caused by the competition between the Taylor rarefaction waves and the SWACER (Shock Wave Amplification due to Coherent Energy Release). Gordon and Sivashinsky [14] assumed that the velocity decrease of detonation in rough tubes is caused by the obstacle drag, and they introduced a drag function into the source term of the momentum equation to model quasi-detonation. However, their model failed in simulating all the complexity of the quasidetonation process and was believed to capture the physical phenomena only in some average sense. More importantly, none of the above points of view can interpret the precipitate transition from detonation to quasidetonation. Therefore, the mechanism of detonation to quasi-detonation transition is still unknown now.

The motive of this study is to explore the mechanism of transition from detonation to quasi-detonation. In the first part, the thermodynamic properties of quasi-detonation are analyzed. It is confirmed that the quasi-detonation is caused by thermal choking. In the second part, a new physical model is proposed to simulate the flow field after thermal choking. One-dimensional numerical simulation is conducted and quasi-detonation is firstly obtained. In the third part, the numerical results are discussed and compared with existing experimental observations. In the last part, the conclusion of this study is given.

\section{ANALYSIS OF THERMAL CHOKING CONDITION}

In the constant area duct, the chemical release of energy may cause thermal choking of the supersonic flow in the duct. This occurs when sufficient thermal energy is added to the supersonic flow in the duct to reduce the flow Mach number to unity. The flow is then unable to accept additional thermal energy. Any attempt to further add energy results in a reduction of the mass flow through the duct, and a consequential spilling of surplus mass flow from the duct entrance. The flow is then said to be thermally choked.

The limit of maximum energy release to the flow in a constant area duct is given by the function of the free stream Mach number $\mathrm{M}_{1}$ and the total enthalpy $C_{\mathrm{p}} T_{01}[15]$.

$$
\frac{(\Delta Q)_{\max }}{C_{p} T_{01}}=\left[\frac{1+\gamma M_{1}^{2}}{(1+\gamma) M_{1}}\right]^{2}\left[\frac{1+\gamma}{2+(\gamma-1) M_{1}^{2}}\right]-1
$$

By simple analysis, we can confirm that $\mathrm{CJ}$ detonation has just reach the thermal choking condition because the Mach number of the products relative to the detonation front is unity by classical CJ theory. For example, for the stoichiometric $\mathrm{H}_{2} /$ Air mixture, the gas constant is $R=368.9 \mathrm{~J} / \mathrm{kg} / \mathrm{K}$, the specific heat ratio $\gamma=1.29$, the constant pressure specific heat capacity $C_{\mathrm{p}}=1640 \mathrm{~J} / \mathrm{kg} / \mathrm{K}$, the initial temperature $T_{0}=300 \mathrm{~K}$, and the initial pressure $P_{0}=1 \mathrm{~atm}$, respectively. The CJ detonation velocity is $D_{\mathrm{CJ}}=1950 \mathrm{~m} / \mathrm{s}$, the Mach number of CJ detonation $M_{\mathrm{CJ}}=4.8$, and the total enthalpy of the free stream $H_{0}=C_{\mathrm{p}} T_{0}=2.8 \times 10^{6} \mathrm{~J} / \mathrm{kg}$, respectively. According to Eq.(1), the ratio of the maximum heat release to the total enthalpy is 1.0 , thus the maximum heat release to the supersonic system is limited to $\Delta Q_{\max }=2.8 \times 10^{6} \mathrm{~J} / \mathrm{kg}$. The specific heat release of the one-step detonation model for stoichiometric $\mathrm{H}_{2} /$ Air mixture is $q=2.72 \times 10^{6} \mathrm{~J} / \mathrm{kg}$ [16]. Making some notional allowance for the approximate nature of the analysis, it can be concluded that thermal choking just occurs to the $\mathrm{CJ}$ detonation.

By the same analysis progress, we can find that quasi-detonation is thermally choked. For the same chemical reaction system, the velocity of quasi-detonation is about $D_{\text {quasi }}=1050 \mathrm{~m} / \mathrm{s}, M_{\text {quasi }}=2.7$, and the total enthalpy 
$H_{\text {quas }}=1.2 \times 10^{6} \mathrm{~J} / \mathrm{kg}$, respectively. According to Eq.(1), the ratio of the maximum heat release to the total enthalpy is 0.57 , thus the maximum heat release to the supersonic system is limited to $\Delta Q_{\text {max,quasi }}=0.68 \times 10^{6} \mathrm{~J} / \mathrm{kg}$, which is much lower than the specific heat release of detonable reactants. So, for the quasi-detonation, the flow field is already thermally choked and some self-adjustment to a new flow field will occur.

The classical CJ theory does not tell us what will happen after the flow field is thermally choked. We postulate that when the heat release to the flow filed is beyond the maximum heat calculated by Eq.(1), the flow filed becomes unstable and will change to a new quasi-stable state precipitately by itself to satisfy the limit condition of maximum heat addition. A large amount of energy is needed to support this self-adjustment process. This means that this part of energy does not support the propagation of the detonation any more. On this hypothesis, we propose a new physical model to simulate the quasi-detonation. And one-dimensional numerical simulate is conducted by using this new physical model in the following parts.

\section{GOVERNING EQUATIONS AND PHYSICAL MODEL}

The one-dimensional governing equations for $\mathrm{CJ}$ detonation is as follows. Viscous terms and diffusive effects are neglected because of their minor roles in determining the overall flow dynamics of detonation. The chemical reaction model is the classical one-step overall Arrhenius type chemical reaction kinetics.

$$
\begin{gathered}
\frac{\partial U}{\partial t}+\frac{\partial F}{\partial x}=S \\
U=\left(\begin{array}{c}
\rho \\
\rho u \\
\rho e \\
\rho Z
\end{array}\right) F=\left(\begin{array}{c}
\rho u \\
\rho u^{2}+p \\
(\rho e+p) u \\
\rho u Z
\end{array}\right) S=\left(\begin{array}{c}
0 \\
0 \\
0 \\
\dot{\omega}
\end{array}\right) \\
\dot{\omega}=-K \rho Z \exp \left(-E_{\mathrm{a}} / R T\right) \\
e=\frac{p}{(\gamma-1) \rho}+u^{2} / 2+Z q
\end{gathered}
$$

where, $\rho, u, e$, and $Z$ are density, velocity, specific total energy, and chemical reaction progress parameter (i.e., the mass fraction of reactants), respectively. In Eq.(4), $\omega$ is the mass production rate of products, $K$ is the pre-exponential factor, $T$ is the temperature in Kelvin $\mathrm{K}$, and $E_{\mathrm{a}}$ is the activation energy per unit mass of reactants, respectively. In the energy conservative equation of Eq.(5), the first term of the right hand is the internal energy, the second term is the kinetic energy, and the third term is the chemical reaction heat release.

Considering the above discussions on quasi-detonation, we introduce a new term $\varphi$ into the energy equation to represent the part of energy which supports the transition process from CJ detonation to quasi-detonation. And we further postulate that this term $\varphi$ is proportional to the local kinetic energy of the flow field.

$$
\begin{gathered}
e=\frac{p}{(\gamma-1) \rho}+u^{2} / 2+Z q+\phi \\
\phi=c u^{2} / 2
\end{gathered}
$$

By combining Eqs.(6) and (7), we obtain the new energy equation of Eq.(8) for quasi-detonation. In this new model, the energy dissipation function $C$ represents the portion of energy which is used to support the flow field selfadjustment after it is thermally choked. It is clearly seen that $C=1$ is for CJ detonation. At the present time, we do not know the exact function of parameter $C$, but we can firstly study the influence of $C$ on the flow field properties by varying its value in numerical simulations to see whether quasi-detonation will occur.

$$
e=\frac{p}{(\gamma-1) \rho}+C u^{2} / 2+Z q
$$

One-dimensional numerical simulation was conducted. The computational domain is taken to be a straight detonation tube with the left end closed and the right end open. The tube is $0.5 \mathrm{~m}$ long, which is fully filled with the premixed stoichiometric $\mathrm{H}_{2} /$ Air mixture at $1 \mathrm{~atm}$ and $300 \mathrm{~K}$. The parameters for this model are $Z=1.0, \gamma=1.29$, 
$R=368.9 \mathrm{~J} / \mathrm{kg} / \mathrm{K}, q=2.72 \times 10^{6} \mathrm{~J} / \mathrm{kg}, E_{\mathrm{a}}=4.794 \times 10^{6} \mathrm{~J} / \mathrm{kg}, K=7.5 \times 10^{9} \mathrm{~s}^{-1}$, respectively [16]. Detonation is initiated by smaller region of reactants with high pressure and temperature specified near the closed end, and then it propagates from left to right. ENO scheme and TVD Runge-Kutta method are applied [17]. The convective flux is split by Steger-Warming flux splitting method [18]. The uniform grid spacing is $d x=0.1 \mathrm{~mm}$. The mirror reflection boundary conditions are applied on the closed end.

\section{RESULTS AND DISCUSSION}

By varying the values of parameter $C$ from 1.0 to 3.0 in one-dimensional numerical simulations, we firstly simulated the transition from $\mathrm{CJ}$ detonation to quasi-detonation and obtained the structure of quasi-detonation. Figure 1 shows the detonation propagation speed with different values of parameter $C$. For $C=1.0$, the detonation is CJ detonation, and the propagation speed is $1950 \mathrm{~m} / \mathrm{s}$. As the values of $C$ increase from 1.0 to 2.7 , the detonation speed slowly decreases to $1800 \mathrm{~m} / \mathrm{s}$, being about 92 percent of CJ detonation speed. Chao et al. [6] also observed this kind of detonation propagating with about 90 percent of CJ speed in experiments, and they called it "sub-CJ detonation".

When we continue to increase the value of $C$ to 2.8 , a great change of the flow field occurs. The detonation speed precipitately decreases to about $1050 \mathrm{~m} / \mathrm{s}$, and then keeps constant. The Mach number of quasi-detonation is $M_{\text {quasi }}=2.78$. This speed is about 53 percent of CJ speed, which is equal to the speed of quasi-detonation. It can be thought that $C=2.8$ is a critical condition. Under this condition, transition from $\mathrm{CJ}$ detonation to quasi-detonation occurs precipitately. It can also be estimated for $C=2.8$ that nearly 65 percent of energy is absent from the detonation propagation, which is close to the fact that nearly 75 percent of chemical reaction energy is lost in quasi-detonation discussed above.

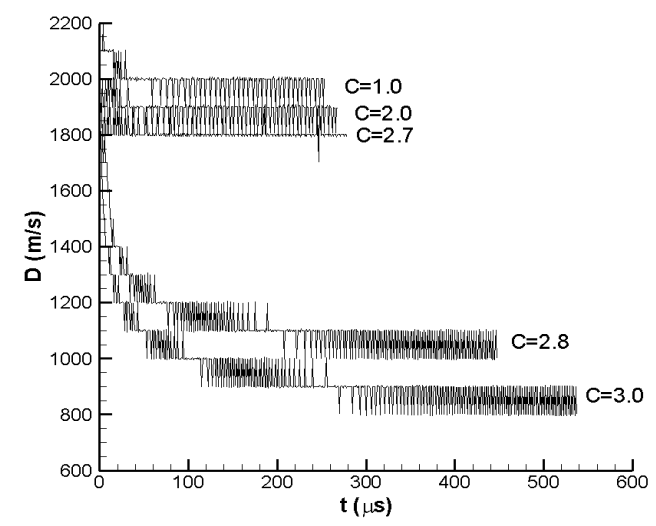

FIGURE 1. Quasi-detonation Speed Variations as the Energy Dissipation Parameter C Varies from 1.0 to 3.0

The pressure profiles of $\mathrm{CJ}$ detonation, sub-CJ detonation, and quasi-detonation with different values of parameter $C$ are plotted in Fig.2. From Fig.2(a) we can see that for $C<2.8$, these pressure profiles at the same instant are very similar. The detonation front is followed by rarefaction waves, and the point of zero velocity is nearly at the center point between the detonation front and the closed end. The only difference is that the sub-CJ detonation front is behind the CJ detonation front at the same instance because the speed of sub-CJ detonation is slower than CJ detonation speed. This means that the strength of rarefaction wave behind sub-CJ detonation is stronger than that of CJ detonation. This result demonstrates that CJ detonation and sub-CJ detonation have the same mechanism qualitatively.
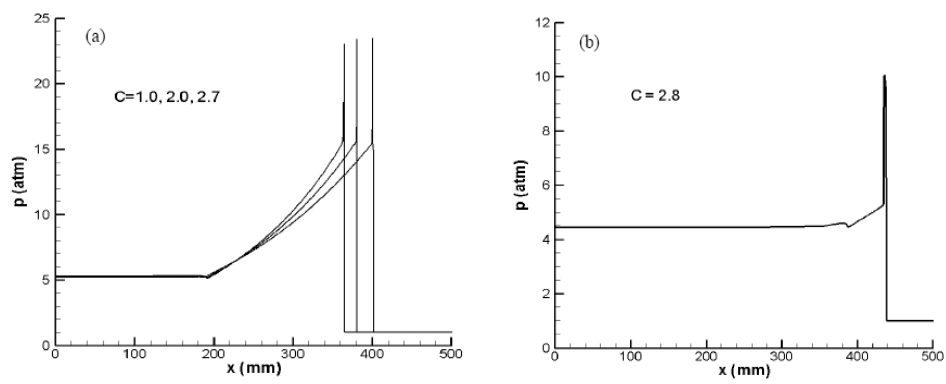

FIGURE 2. Pressure Profiles of CJ and Quasi-detonation under Different Values of Energy Dissipation Parameter C 
But the profile of quasi-detonation in Fig.2(b) is completely different from that of CJ detonation. Firstly, the length of rarefaction region is very short, and the zero velocity point is very close to the quasi-detonation front instead of at the middle point. Secondly, the pressure at and behind the front is much lower than that of CJ detonation. For instance, the pressure at the $\mathrm{CJ}$ detonation front is about 25atm, while the pressure at the quasi-detonation front is about 9 atm. This pressure of $9 \mathrm{~atm}$ is just the pressure behind a normal shock wave of $M a=2.78$. This result indicates that quasi-detonation and CJ detonation have different aerodynamic and thermodynamic properties, and thus must have different mechanism.

In order to further study the structure of quasi-detonation front, the profiles of temperature and chemical reaction progress parameter $Z$ for quasi-detonation at $C=2.8$ are plotted in Fig.3. The position of parameter $Z$ decreasing from 1.0 to 0.0 indicates the position of the flame front. From this figure we can find that the quasi-detonation front is composed of a leading shock wave and a flame front. There two discontinuities, one is the leading shock wave, and the other is the combustion flame front. This double-discontinuity front structure is consistent with experimental observations [10].

The flow field is divided into three regions by these two discontinuities, i.e., the region 0 in front of the leading shock wave, the region 1 between the shock and the flame front, and the region 2 behind the flame front. In region 1 , the temperature first increases and then decreases to a much lower value. The thermodynamic properties of region 1 are not the states behind a normal shock wave. Thus it is not of a CJ type and cannot be interpreted by the classical $\mathrm{CJ}$ theory. This result is consistent with the discussion in the reference [7].

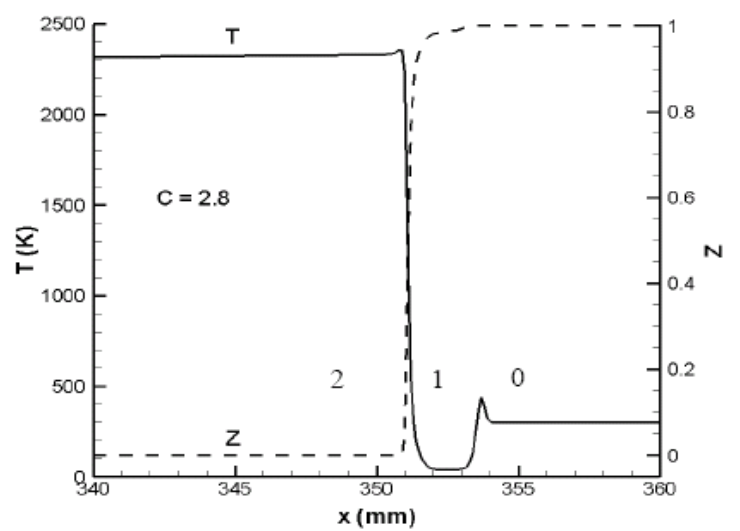

FIGURE 3. Profiles of Temperature and Chemical Reaction Progress Parameter $Z$ for Quasi-detonation at $C=2.8$

Figure 4 shows the profile of the gas Mach number relative to the quasi-detonation front. The flame front is also indicated by the dashed line of parameter $Z$ in this figure. It can be seen that the Mach number in region 0 is $\mathrm{M}_{0}=2.78$. The flow in region 1 behind the leading shock wave becomes subsonic, $M_{1}<1.0$. The Mach number just in front of the flame front is less than 0.1. According to Eq.(1), the maximum heat addition to a stationary system is infinite. Behind the flame front, the gas is then accelerated to sonic, $\mathrm{M}_{2}=1.0$. This result reveals a new mechanism that when the system is thermally choked, a "pseudo-shock" is produced in front of the flame front. The pseudo-shock changes the supersonic flow to subsonic flow in order to increase the maximum energy addition to the combustion system to satisfy the condition of Eq.(1). When this condition is satisfied by self-adjustment of the flow field, a quasi-steady condition of quasi-detonation is reached. We call the leading shock wave pseudo-shock here because the thermodynamic properties behind the pseudo-shock are different from that behind a normal shock wave. This result is consistent with the hypothesis made in the physical model. 


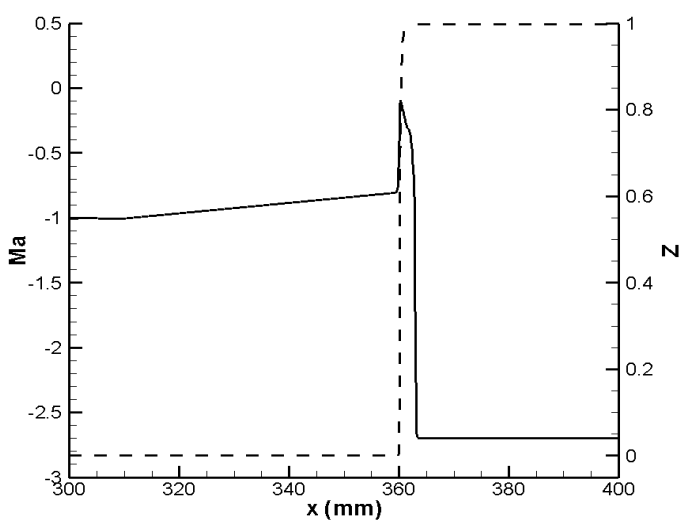

FIGURE 4. Profiles of Relative Mach number and Reaction Parameter $\mathrm{Z}$ for Quasi-detonation at $C=2.8$

Other parameters such as density, velocity, and sound speed are also in good agreement with experimental observations qualitatively. They are not included in this paper because of the length limitation. They will be discussed in the subsequent papers.

\section{CONCLUSIONS}

In this paper, we first confirmed that quasi-detonation is of thermal choking by theoretical analysis and numerical simulation. Thermal choking makes the flow field change to a new quasi-steady state, where the leading shock wave, the chemical reaction heat release, and the maximum heat addition to the system reach a new equilibrium condition. Some amount of energy is consumed in this transition process instead of supporting the detonation propagation. A new physical model is proposed for quasi-detonation, in which the energy loss caused by supersonic combustion is considered. One-dimensional simulation is conducted and quasi-detonation is first obtained. The numerical results are in good agreement with previous experimental observations qualitatively, indicating that both the mechanism discussed and the physical model proposed for quasi-detonation is physically correct.

\section{ACKNOWLEDGMENTS}

This research was supported by the National Natural Science Foundation of China (Grant No.10632090).

\section{REFERENCES}

1. G. Dupre, O. Peraldi, J.H.S. Lee, and R. Knystautas, Progr. Astronaut. Aeronaut. 114, 248-263, (1988).

2. J.H.S. Lee, R. Knystautas, C.K.Chan, Proc. Combust. Inst., 20, 1663-1672, (1985).

3. A. Teodorcyzk and J.H.S. Lee, Shock Waves 4, 225-236, (1995).

4. M.I. Radulescu and J.H.S. Lee, Combust. Flame 131, 29-46, (2002).

5. R.S. Chue, J.H.S. Lee, T. Scarinci, A. Papyrin, and R. Knystautas, Progr. Astronaut. Aeronaut. 153, 270-282(1993).

6. J. Chao and J.H.S. Lee, Shock Waves 12, 277-289, (2003).

7. Yujian Zhu, Jiming Yang, and J.H.S. Lee, Explosion and Shock Wave 28(2), 98-104, (2008). (in Chinese)

8. H.G. Wagner, "Some Experiments about Flame Acceleration," in Proceedings of first International Specialist Meeting on Fuel-Air Explosions, Montreal, pp. 77-99, (1982).

9. J.H.S. Lee, "The propagation of Turbulent Flame and Detonations in Tubes," in Advances in Chemical Reaction Dynamics, edited by P.M. Rentzepis and C. Capellos, Kluwer Academic Publishers, pp. 346-387, (1986).

10. A.K. Oppenheim, "Gasdynamic Analysis of the Development of Gaseous Detonation and its Hydraulic Analogy," in Proc. of Fourth Symp., (Intl.) on Combustion, Baltimore, pp. 471-480, (1953).

11. K.I. Shchelkin and Y.K. Troshin, NASA TT F-231, (1964).

12. D.H. Edward et al., Journal of Physics D: Applied Physics 11, 2103-2217, (1978).

13. R.S. Chue, "High Speed Deflagration and its Transition to Detonation," Ph.D Thesis, McGill University, 1993.

14. P.V. Gordon and G.I. Sivashinsky, Combust. Flame 136, 440-444, (2004).

15. B.G. Tong, X.Y. Kong, and G.H. Deng, Aerodynamics, Beijing: Higher Education Press, 1996, pp. 104-106. (in Chinese)

16. F. Ma, Y.J. Choi, and V. Yang, J. Propul. Power 21, 512-526, (2005).

17. C.W. Shu and S. Osher, J. Comput. Phys. 83, 32-78, (1989).

18. J.L. Steger and R.F. Warming, J. Comput. Phys. 40, 263-293, (1981). 